\title{
Outcome heterogeneity and bias in acute experimental spinal cord injury
}

\author{
A meta-analysis
}

Ralf Watzlawick, MD, Ana Antonic, PhD, Emily S. Sena, PhD, Marcel A. Kopp, MD, Julian Rind, MS, Ulrich Dirnagl, MD, Malcolm Macleod, MD, David W. Howells, PhD, ${ }^{*}$ and Jan M. Schwab, MD, PhD*

Neurology ${ }^{\circledR}$ 2019;93:e40-e51. doi:10.1212/WNL.0000000000007718

\author{
Correspondence \\ Dr. Schwab \\ jan.schwab@osumc.edu
}

\section{Abstract \\ Objective}

To determine whether and to what degree bias and underestimated variability undermine the predictive value of preclinical research for clinical translation.

\section{Methods}

We investigated experimental spinal cord injury (SCI) studies for outcome heterogeneity and the impact of bias. Data from 549 preclinical SCI studies including 9,535 animals were analyzed with meta-regression to assess the effect of various study characteristics and the quality of neurologic recovery.

\section{Results}

Overall, the included interventions reported a neurobehavioral outcome improvement of $26.3 \%$ (95\% confidence interval 24.3-28.4). Response to treatment was dependent on experimental modeling paradigms (neurobehavioral score, site of injury, and animal species). Applying multiple outcome measures was consistently associated with smaller effect sizes compared with studies applying only 1 outcome measure. More than half of the studies (51.2\%) did not report blinded assessment, constituting a likely source of evaluation bias, with an overstated effect size of $7.2 \%$. Assessment of publication bias, which extrapolates to identify likely missing data, suggested that between $2 \%$ and $41 \%$ of experiments remain unpublished. Inclusion of these theoretical missing studies suggested an overestimation of efficacy, reducing the effect sizes by between $0.9 \%$ and $14.3 \%$.

\section{Conclusions}

We provide empirical evidence of prevalent bias in the design and reporting of experimental SCI studies, resulting in overestimation of the effectiveness. Bias compromises the internal validity and jeopardizes the successful translation of SCI therapies from the bench to bedside.

\footnotetext{
*These authors contributed equally to this work.
}

From Charité-Universitätsmedizin Berlin (R.W., M.A.K., J.R., U.D., J.M.S.), corporate member of the Freie Universität Berlin, Humboldt-Universität zu Berlin, and Berlin Institute of Health; Department of Neurology and Experimental Neurology (R.W., M.A.K., J.R., J.M.S.), Charité Campus Mitte, Clinical and Experimental Spinal Cord Injury Research Laboratory (Neuroparaplegiology), Charité-Universitätsmedizin Berlin; Department of Neurosurgery (R.W.), Freiburg University Medical Center, Germany; Department of Neuroscience (A.A.), Central Clinical School, Monash University, Melbourne; Stroke Division (E.S.S., M.M., D.W.H.), Melbourne, Victoria, Australia; Departments of Neurology and Clinical Neurosciences (E.S.S., M.M.), University of Edinburgh, UK; Center for Stroke Research Berlin (U.D.) and Excellence Cluster Neurocure (U.D.), Charité-Universitätsmedizin, Berlin, Germany; German Center for Neurodegenerative Diseases (U.D.), Bonn; Berlin Institute of Health (M.A.K., U.D.), Germany; University of Tasmania (D.W.H.), School of Medicine, Faculty of Health, Medical Sciences Precinct, Hobart, Australia; Department of Neurology (J.M.S.), Spinal Cord Injury Medicine (Paraplegiology), and Belford Center for Spinal Cord Injury (.M.S.), Departments of Neuroscience and Physical Medicine and Rehabilitation, The Neurological Institute, The Ohio State University, Wexner Medical Center, Columbus.

Go to Neurology.org/N for full disclosures. Funding information and disclosures deemed relevant by the authors, if any, are provided at the end of the article. 


\section{Glossary}

BBB = Basso, Beattie, Bresnahan; BMS = Basso Mouse Scale for Locomotion; CAMARADES $=$ Collaborative Approach to Meta-Analysis and Review of Animal Data From Experimental Studies; CI = confidence interval; OEG = olfactory ensheathing glia; SCI = spinal cord injury.

Traumatic spinal cord injury (SCI) affects hundreds of thousands of patients worldwide every year, and it is estimated that up to 5 million patients are currently affected by the chronic consequences of SCI. ${ }^{1,2}$ Despite successful improvements in the prevention of traumatic spinal cord injuries, it has been predicted that the growing population will result in a stable or even an increased total number of patients with SCI. ${ }^{3}$ However, limited financial resources, heterogeneous lesions, and small numbers of clinical trials impair clinical translation. Finding new treatments for these individuals relies on animal models of SCI. These are believed to have good face validity and to be sufficiently like SCI in humans to make them highly relevant for translation. ${ }^{4}$ This holds true as long as known translational limitations are taken into account. These include differences in locomotion (quadrupedal locomotion in rodent mod$\mathrm{els}^{5}$ ), neural innervation relevant for locomotion or fine motor control, ${ }^{6,7}$ different injury paradigms (sustained cord compression, ${ }^{8}$ concomitant injury of nerve roots in human $\mathrm{SCI}^{5}$ ), inflammatory responses, ${ }^{9}$ and other aspects of the physiology and pathophysiology of experimental SCI in rodents. In addition to cross-species differences, methodologic shortcomings are also believed to contribute to translational failure (translational roadblock) of animal model-derived findings. ${ }^{10-12}$ To determine the extent to which avoidable methodologic limitations apply to experimental SCI research, we aimed to characterize the impact of study design, study quality, and bias. To do so, we have performed a meta-analysis of published data from SCI animal studies. These techniques allowed us to interrogate data from larger numbers of animals, reducing the effect of individual underpowered studies. Insufficient statistical power has been identified as a major independent shortcoming limiting the translational and predictive value of animal models. ${ }^{13,14}$

The medical need for developing new therapies emphasizes the necessity for preclinical animal studies to generate clinically valuable (translational) data. This requires the assessment of confounding variables, which might impair the signal-to-noise ratio and reduce the likelihood of detecting an effect.

Risk of experimental and publication bias limits the value of the published literature on drug testing because both lead to overestimation of effect size. Given that systematic reviews and meta-analyses have identified limited internal validity (e.g., due to bias) associated with inflated effect sizes in preclinical ischemic brain injury studies, ${ }^{12}$ we investigate whether and to what degree preventable bias also influences experimental SCI studies. The pragmatic Collaborative Approach to MetaAnalysis and Review of Animal Data From Experimental Studies (CAMARADES) approach has value in determining the likelihood that a particular dataset is subject to these problems.

In this meta-analysis and meta-regression, we determine the impact of study characteristics, methodologic and reporting bias, and common meta-epidemiologic characteristics on neurobehavioral recovery after experimental SCI in a large cohort of 9,535 animals.

\section{Methods}

\section{Literature search}

This study includes data stored in the CAMARADES database. The CAMARADES database contains preclinical animal data for various diseases (e.g., stroke, SCI, myocardial infarction, neuropathic pain) hosted at the University of Edinburgh since 2004. Access to the CAMARADES database is internationally available on request. For this study, all available SCI animal data were selected from the database in September 2015, including 6 independently assessed literature analyses of different SCI interventions: decompression, hypothermia, pharmaceutical inhibitors of the Rho/ROCK pathway, cellular treatment (stem cells, olfactory ensheathing glia [OEG]), and physical exercise after the injury. All of the corresponding study protocols and a protocol of this metaanalysis were finalized in advance of any data collection and are accessible online (dcn.ed.ac.uk/camarades/research. html\#protocols). Five of those 6 studies have already been published as systematic reviews and meta-analyses. ${ }^{15-20}$ Eligibility criteria differ moderately among the included studies, but each reported locomotor recovery in experimental SCI models. We excluded studies that did not report the number of animals in each group, the mean effect size, and its variance. The initial literature searches in 3 online databases (PubMed, EMBASE, and ISI Web of Science) were performed until October 2014 for studies published before then and were screened by 2 independent investigators. The searches within the databases were not restricted to specific years.

\section{Data extraction}

Study characteristics from each publication were extracted into the CAMARADES database as outlined in previous reviews. ${ }^{15-20}$ In brief, when a single publication reported $>1$ experiment, they were treated as independent experiments. 
For experiments reporting the improvement of a functional outcome measured by a neurobehavioral score, only the final time points were extracted. Studies were excluded if they did not report the number of animals in each group, the mean effect size and its SD, or SEM. Where various outcome measurements were reported for the same group of animals, we pooled those containing a value for multiple scales. The quality of each study was assessed with a modified 9-point checklist $^{21}$ (1) reporting of a sample size calculation, (2) control of the animals' temperature, (3) use of anesthetics other than ketamine (because of its marked intrinsic neuroprotective activity), (4) randomized treatment allocation, (5) treatment allocation concealment, (6) blinded assessment of outcome, (7) publication in a peer-reviewed journal; (8) statement of compliance with regulatory requirements, and (9) statement of potential conflicts of interest.

\section{Analysis}

The normalized effect size (normalized mean difference) was calculated for each experimental comparison as the proportional improvement in the treated compared to the control group, along with a standard error of this estimate. When a single control group served multiple treatment groups, the size of the control group was adjusted to account for this. This corrected number was used for any further calculation and in the weighting of effect sizes. ${ }^{22}$

We stratified the analysis according to (1) the applied treatment, (2) the neurobehavioral score used (Basso, Beattie, Bresnahan $[\mathrm{BBB}]$ score $^{23}$; Basso Mouse Scale for Locomotion $[\mathrm{BMS}]^{24}$; Tarlov scale ${ }^{25}$; others), (3) the model and level of the injury, (4) study characteristics (time to treatment, time of outcome assessment, animal sex and strain), (5) anesthetic regimen (anesthetic used and method of ventilation), and (6) methodologic quality of the studies according to the 9-point checklist. Cohorts within a stratification accounting for $>1 \%$ of the total animal number were considered an individual group. Smaller cohorts accounting for $<1 \%$ were grouped together and referred to as others.

We applied random-effect meta-analysis regression to calculate an overall treatment effect, combining efficacy across studies to assess significant differences between the groups for each stratification criteria. Meta-regression with the metareg function of STATA/SE13 (StataCorp, College Station, TX) was used, $^{26}$ and the significance level was set at $p=0.05$. The metaregression was performed in a univariate manner because major concerns about the reliability of multivariate analysis have been raised with the need for further statistical investigation. ${ }^{27}$ Adjusted $R^{2}$ values were calculated to assess how much residual heterogeneity was explained by each independent variable within a stratified dataset. ${ }^{22}$ The adjusted $R^{2}$ is defined as the ratio of explained variance of the respective stratification to total variance (of all experiments together). Higher $R^{2}$ values are indicative of a higher explanatory value of this stratification. Restricted maximum likelihood was used to estimate the additive (between-study) component of variance tau 2 within the meta-regression. ${ }^{26}$ The dependent variable was, in all cases, the normalized effect size.

Funnel plots ${ }^{28}$ and trim and fill analyses ${ }^{29,30}$ were performed with the metatrim function of STATA/SE13. The Duval and Twee$\mathrm{die}^{29}$ nonparametric trim and fill method estimates the number of missing studies (publication bias) that might have been performed but are not available for a meta-analysis using simple rankbased data augmentation techniques adjusted for missing studies. Normalized mean difference (effect size) was plotted against precision (1/SEM). The metabias function detects the presence of publication bias by Egger regression measured by the intercept from standard normal deviates against precision. ${ }^{31}$ Figures were drawn with SigmaPlot (Systat Software Inc, San Jose, CA).

\section{Data availability}

Data not provided in the article will be shared at the request of other investigators for purposes of replicating procedures and results.

\section{Results}

\section{Selection of datasets and study details}

The CAMARADES database contained details on 549 experiments from 303 included publications from 1967 to 2014 reporting functional improvement assessed by neurobehavioral scores (table 1 ).

We included data from 9,535 animals. Overall, the number of control animals used was $20 \%$ smaller than the number that received treatment (control animals $\mathrm{n}=3,765,39.5 \%$; treated animals $n=5,770,60.5 \%)$. Six different animal species were used to investigate therapeutic interventions for SCI: 8,108 rats $(85 \%), 1,199$ mice $(12.6 \%), 107$ dogs (1.1\%), 57 monkeys $(0.6 \%), 44$ rabbits $(0.5 \%)$, and 20 sheep $(0.2 \%)$.

\section{Study characteristics and their impact on neurobehavioral outcome}

Random-effect meta-regression revealed an overall effect size of $26.3 \%$ ( $95 \%$ confidence interval [CI] 24.3-28.3, $I^{2}=$ 98.6\%) when all included neurobehavioral scores were combined. To explore sources of heterogeneity, we applied metaregression and identified several study design characteristics accounting for significant within- and between-study heterogeneity (figures 1 and 2). Study characteristics are represented in terms of prevalence (width of the bar indicating the number of studies) and variability (CI).

\section{Animal model}

Experimental SCI in rats constituted the most prevalent injury paradigm and outnumbered mouse SCI, even though the latter offers the advantage of genetic manipulation (figure 1A). Variability as indicated by $95 \%$ CIs was larger in mice compared to rats. Effect sizes for larger animal SCI models, including dogs, monkeys, rabbits, and sheep, applied mostly customized scoring systems and were generally larger and linked to greater variability $\left(R^{2}=8.0 \%, p<0.0001\right)$. 
Table 1 Characteristics of different interventions included in the overall analysis

\begin{tabular}{|c|c|c|c|c|c|c|c|}
\hline & Decompression & Exercise & Hypothermia & OEG & $\begin{array}{l}\text { Rho/ROCK } \\
\text { inhibitors }\end{array}$ & Stem cells & Overall \\
\hline Publications, n (\%) & $21(6.9)$ & $25(8.3)$ & $16(5.3)$ & $51(16.8)$ & $14(4.6)$ & $176(58.1)$ & 303 \\
\hline Experiments, $\mathrm{n}(\%)$ & 79 (14.4) & $36(6.6)$ & $25(4.6)$ & $62(11.3)$ & $30(5.5)$ & $317(57.7)$ & 549 \\
\hline Animals, n (\%) & $873(9.2)$ & $575(6.0)$ & $448(4.7)$ & $1,164(12.2)$ & $655(6.7)$ & $5,820(61.0)$ & 9,535 \\
\hline BBB scale, n (\%) & $246(3.4)$ & $498(6.8)$ & $218(3.0)$ & $1,027(14.0)$ & $524(7.2)$ & $4,811(65.7)$ & 7,324 \\
\hline BMS score, $\mathbf{n}(\%)$ & 0 & 55 (12.6) & 0 & 0 & $131(30.0)$ & $250(57.3)$ & 436 \\
\hline Multiple, n (\%) & $282(15.1)$ & $398(21.4)$ & $101(5.4)$ & 0 & 0 & $1,083(58.1)$ & 1864 \\
\hline Inclined plane, n (\%) & $295(90.2)$ & 0 & $32(9.8)$ & 0 & 0 & 0 & 327 \\
\hline Tarlov scale, n (\%) & $27(8.6)$ & 0 & $88(27.9)$ & 0 & 0 & $200(63.5)$ & 315 \\
\hline Others, n (\%) & $151(23.1)$ & $14(2.1)$ & $99(15.1)$ & 137 (20.9) & 0 & $253(38.7)$ & 654 \\
\hline $\begin{array}{l}\text { Overall effect size, } \\
\text { (\% improvement in } \\
\text { outcome score) }\end{array}$ & $34.2(27.4-41.1)$ & $\begin{array}{l}13.1 \\
(4.9-21.4)\end{array}$ & $\begin{array}{l}29.1 \\
(17.1-41.1)\end{array}$ & $\begin{array}{l}23.6 \\
(17.3-30.0)\end{array}$ & $23.2(14.1-32.4)$ & $\begin{array}{l}27.1 \\
(24.5-29.7)\end{array}$ & $\begin{array}{l}26.3 \\
(24.3-28.4)\end{array}$ \\
\hline
\end{tabular}

Abbreviations: BBB = Basso, Beattie, and Bresnahan; BMS = Basso Mouse Scale; OEG = olfactory ensheathing glia.

Cumulative number of publications, number of experiments, included animals, applied outcome measures, and effect size calculated by meta-regression for all outcome measurements are given. Largest effect sizes relate to spinal decompression surgery and hypothermia. Note that multiple outcome measurements contain experiments assessing various outcome measures for a specific animal group (e.g., BBB score and Tarlov scale).

\section{Neurobehavioral scale}

Multiple neurobehavioral outcome scales were used to assess functional recovery after SCI (figure $1 \mathrm{~B}$ ). The $\mathrm{BBB}$ or $\mathrm{BMS}^{23,24}$ score for locomotion was most commonly applied, accounting for $66.7 \%$ (6,302 of 9,462 animals). The 21-point $\mathrm{BBB}$ score evaluates hind-limb movement and fore/hind-limb coordination in an open field test in rats, whereas the BMS score is adapted to recovery patterns in mice. ${ }^{23,24} \mathrm{BBB}$ and BMS scores reported similar improvement of locomotion within the overall analysis (figure $1 \mathrm{~B}$, red square). $\mathrm{BBB}$ and BMS scores were also associated with the smallest measurement variability (CI). The effect size was lowest in cases when multiple assessments ( $>1$ outcome measurement reported in a study) were used $\left(R^{2}=14.7 \%, p<0.0001\right)$. Animals assessed with the outdated Tarlov scale showed the greatest improvement. Despite considerable differences in SCI lesion neuropathology between rats and mice (cavity formation, inflammatory response), ${ }^{32,33}$ the relative amounts of recovery and responder rates after experimental SCI on mice (BMS) and rats $(\mathrm{BBB})$ were comparable.

\section{Blinded assessment of outcome}

Regarding the hypothesis that lack of reporting on study quality criteria is associated with variations in effect sizes, 2 main quality characteristics revealed statistically significant results: blinding of experiments and whether compliance with animal welfare was reported (figure 1, C and D). Fewer than half $(48.8 \%)$ of the interventional studies reported that they were conducted in a blinded manner. Lack of blinding results in evaluation bias that reduces the internal reliability of the experiment and accounts for significantly inflated effect sizes by $7.2 \%\left(R^{2}=2.6 \%, p=0.001\right)$ (figure $\left.1 C\right)$.

\section{Compliance with animal welfare}

Reported compliance with animal welfare protocols was lacking in $29.5 \%(n=2,815)$ of the studies. Studies lacking reported compliance were associated with 5.6\% larger effect sizes and larger variability (figure 1D). The reporting of statement of compliance $\left(R^{2}=2.0 \%, p=0.015\right)$ and blinding during outcome assessment were significantly associated with different effect sizes, both representing items of the 9-point quality checklist. ${ }^{21,22}$

\section{Impact of the design of experimental SCI studies on neurologic recovery in rodent models}

To address the possibility that differences in effect sizes can be attributed to the distinct scoring systems applied to rodent and large animal models, we performed a subanalysis for a BBB/BMS score only population (figure 2). Similar BBB (rat) and BMS (mouse) effect sizes (figure 1B) ensure sufficient comparability as the basis to study the relevance of distinct experimental designs for neurologic recovery. For the $\mathrm{BBB} / \mathrm{BMS}$ only population, meta-regression calculated an effect size of $24.0 \%$ (95\% CI 21.9-26.2, $I^{2}=98.9 \%$; table 2 ).

\section{Type of treatment}

We compared quantitative data about SCI interventions relevant for clinical SCI ranging from acute care to chronic SCI. These include decompression, hypothermia, pharmaceutical (Rho/ROCK pathway) and cellular treatments (stem cells, OEGs), and rehabilitative interventions (voluntary and forced exercise). Acute care interventions are associated throughout with higher effect sizes compared with rehabilitative interventions such as exercise (figure $2 \mathrm{~A}$ ). Improvement in motor 
Figure 1 Neurologic recovery after SCl (effect size): meta-regression stratifying for animal species, neurobehavioral scores, and study quality
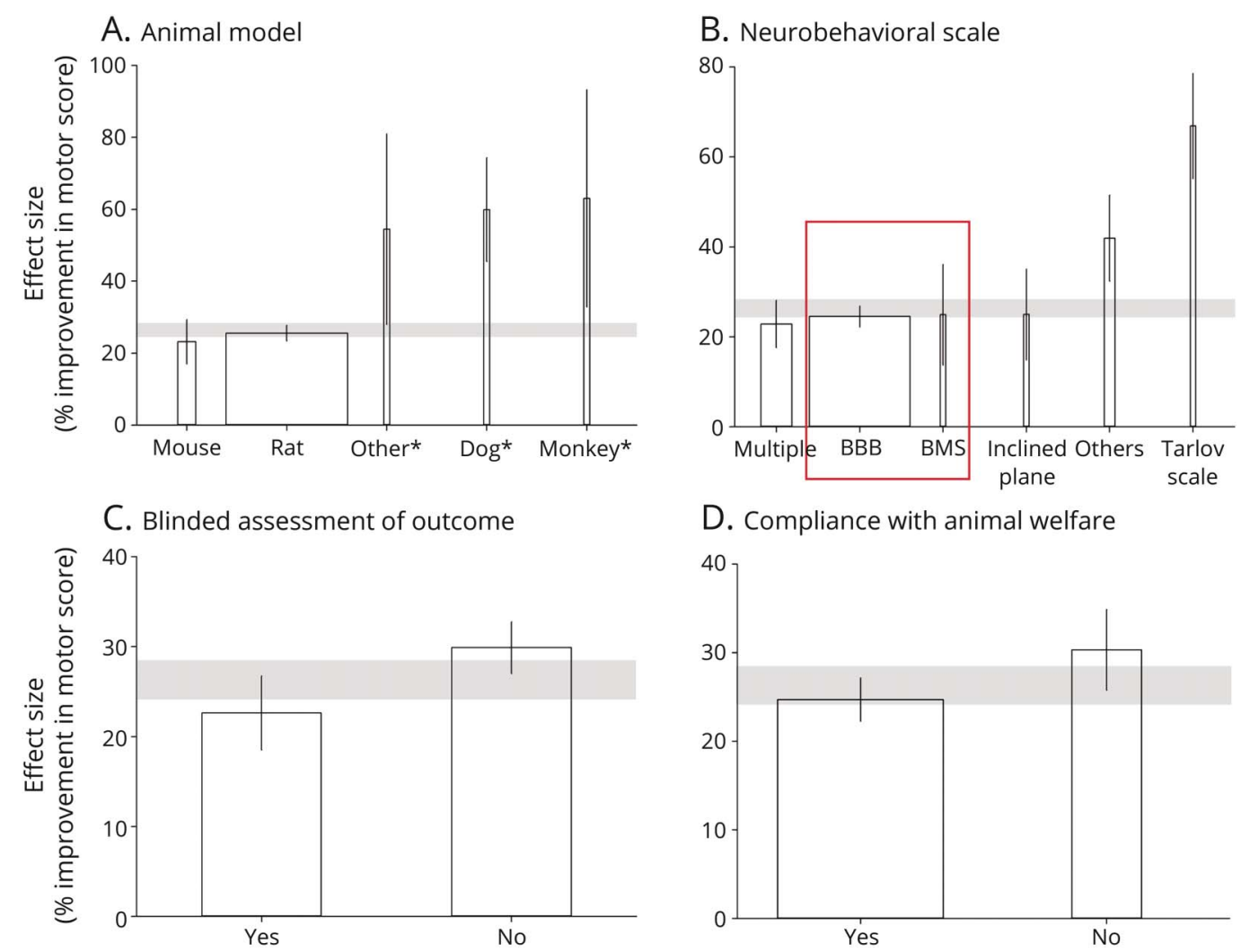

Stratifications of study characteristics within the overall dataset, comprising all reported neurobehavioral outcome scores and animal species. (A) Rat constitutes the most prevalent spinal cord injury SCI model, followed by mice, whereas large animal spinal cord injury (SCI) models, including dogs, monkeys, rabbits, and sheep, are rare and characterized by customized scoring systems with larger effect sizes and extended variability. (B) Various neurobehavioral outcome scales were used to assess functional recovery after SCI in the overall dataset. The Basso, Beattie, and Bresnahan (BBB) score and Basso Mouse Scale (BMS) score for locomotion were identified as the most commonly used scores and reported similar improvement of locomotion. The homogeneous BBB/ BMS only cohort (red square) was assessed in a separate subanalysis. (C) Fewer than half (48.8\%) of the interventional studies reported that they were conducted in a blinded manner, with a difference of $7.2 \%$ in the calculated effect sizes. (D) Reported compliance with animal welfare protocols was lacking in $29.5 \%$ of the studies, which were associated with $5.6 \%$ larger effect sizes and larger variability. Gray bars represent the $95 \%$ confidence interval (CI) of the global estimate (neurologic recovery). Vertical error bars show the $95 \% \mathrm{Cl}$ of individual means. Width of each bar indicates percentage of reported animals number per specified item. Asterisks indicate stratification items for which the bar width was broadened later for better visibility. All stratifications account for a significant proportion of between-study heterogeneity $(p<0.02)$.

outcome after SCI was most pronounced for decompression surgery (27.1\%), followed by stem cells (26.2\%), Rho/ROCK inhibition (21.3\%), and hypothermia (16.3\%). Exercise demonstrated the lowest effect sizes (14.4\%). Stem cell transplantation studies accounted for $65.3 \%$ of the total number of animals $\left(R^{2}=3.0 \%, p=0.0022\right)$ included in this subanalysis.

\section{Level of injury}

The most prevalent lesion level location was Th8, followed by Th9, and Th10 (figure 2B). Level of injury affects the effect size, with closer proximity of the lesion to deafferentated afferent target (in the lumbosacral motor pools) resulting in larger effect sizes, mimicking an exponential curve function $\left(R^{2}=4.5 \%, p=0.0047\right)$. Interpretation of the high cervical SCI data is limited further by small sample size, large variance, and the use of less severe lesion paradigms. Thus, experimental SCI is dominated by low thoracic SCI paradigms.

\section{Sex}

Female rodents outnumbered males (58.2\% females, $27.4 \%$ males; figure $2 \mathrm{C}$ ). Studies including both sexes were characterized by a larger variability resulting in an extended CI $\left(R^{2}=\right.$ $3.5 \%, p=0.0018$ ).

\section{Type of injury}

SCI was induced with 5 different types of injury (figure 2D). Contusion and transection were the most severe injuries and produced the smallest interventional effect. Static compression was associated with slightly better effect sizes, followed by hemisection. Photochemical injuries (focal topical application of high-energy light or chemicals) were rarely applied and induced only minor injury, associated with the largest improvement responses in motor score $\left(R^{2}=3.8 \%, p=\right.$ $0.005)$. Contusion SCI was by far the most prevalent injury paradigm $(\mathrm{n}=3,983$ animals $)$. 


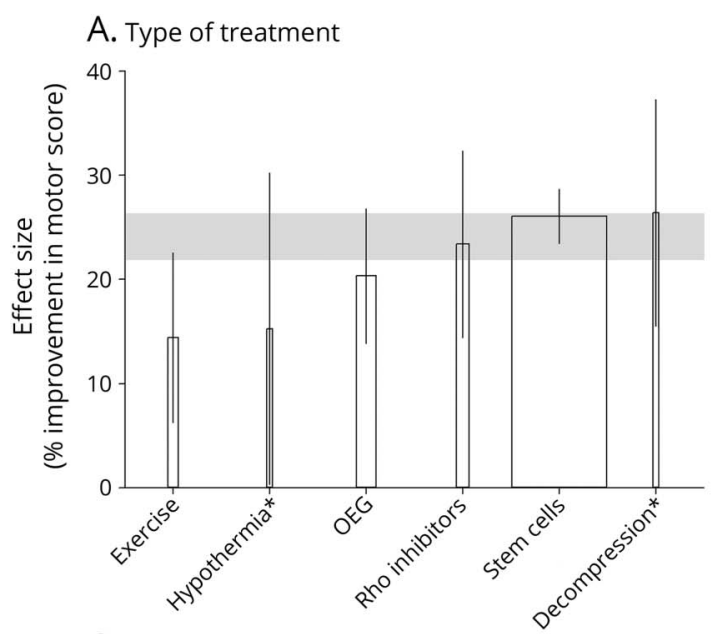

B. Level of injury

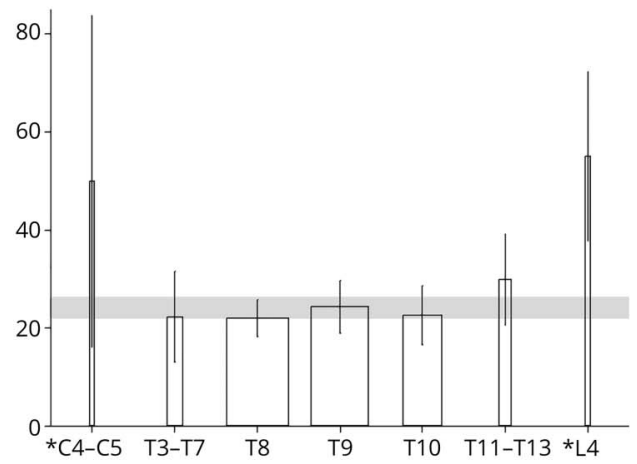

C. Sex of animal

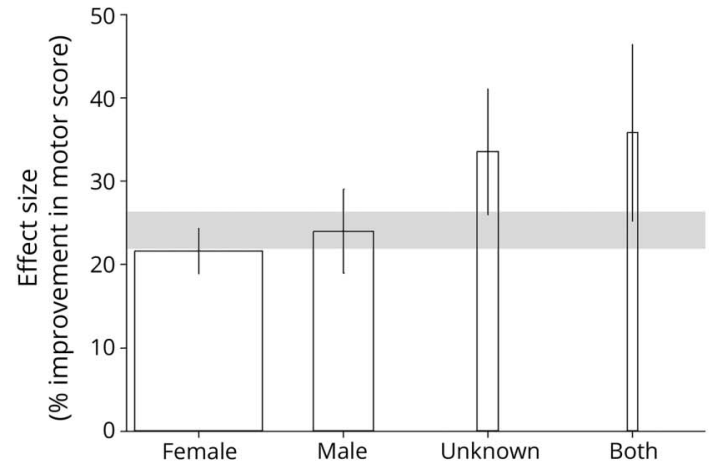

D. Type of injury

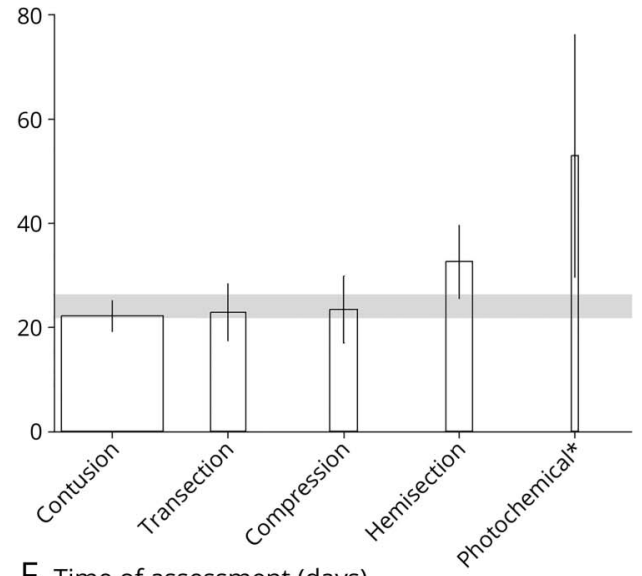

E. Location of experimental laboratory

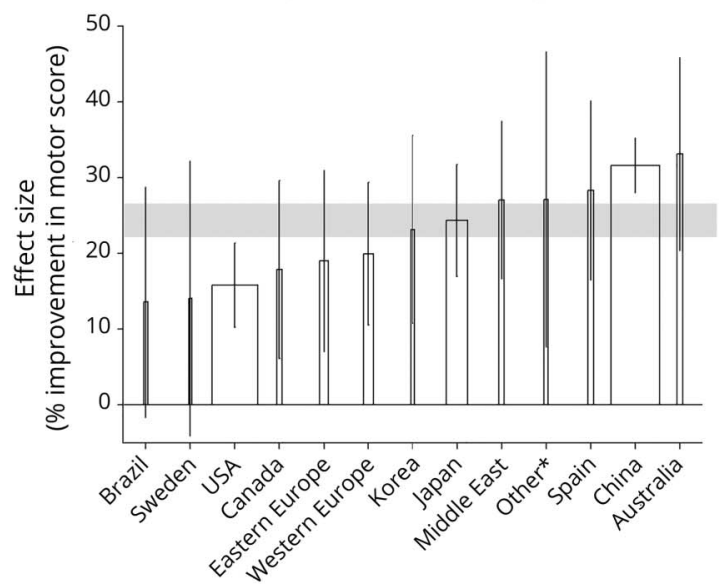

F. Time of assessment (days)

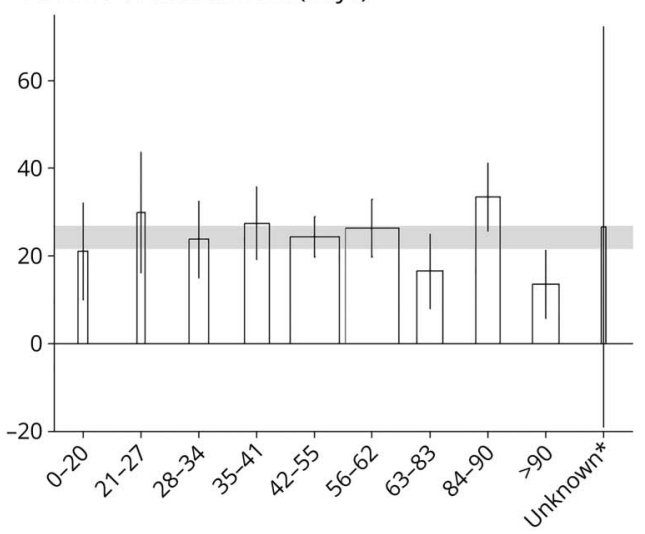

Differential aspects of spinal cord injury (SCI) modeling on neurologic recovery were assessed by including only studies that applied Basso, Beattie, and Bresnahan (BBB) or Basso Mouse Scale (BMS) scores. This homogeneous cohort (red square, B) comprises 6,048 of 9,535 animals (63.4\%). (A) Six different interventions were included in the meta-regression. (B) Lesion-level matter. A more rostral lesion level (high thoracic) along the neuraxis resulted in smaller outcome gain compared to more distal thoracic lesions. High cervical (C2-C4) lesion levels were considered a separate cohort in this regard in that only unilateral hemisections can be performed at those levels because complete transections or severe contusions are technically challenging or will otherwise cause immediate death of the animal. (C) Female rodents constitute the main cohort tested in experimental SCI. (D) Severe contusion and complete transection injuries resulted in the most impaired neurologic function and lowest effect size, followed by static clip compression. Hemisection and photochemical injuries (focal topical application of high-energy light and/or chemicals) induced only minor injury, associated with the largest improvement. (E) Geographic location of the experimental laboratory influenced the amount of reported neurologic recovery. Individual countries were identified if they contributed $>200$ animals to the dataset. (F) Effect size of the investigated intervention decreased with extended assessment period after SCl. Effect size depended on the duration of the assessment and started to intermittently drop below the average with observational time frames $>8$ weeks, whereas the variability remained similar. Gray bars represent the $95 \%$ confidence interval $(\mathrm{Cl})$ of the global estimate (neurologic recovery). Vertical error bars show the $95 \% \mathrm{Cl}$ of individual means. Width of each bar indicates the percentage of reported animal numbers per specified item. Asterisks indicate stratification items for which the bar width was broadened later for better visibility. All stratifications account for a significant proportion of between-study heterogeneity $(p<$ 0.001). $\mathrm{OEG}=$ olfactory ensheathing glia. 
Table 2 Analysis of the BBB/BMS only cohort after correction for missing data (delta inflation)

\begin{tabular}{|c|c|c|c|c|c|}
\hline Intervention & $\begin{array}{l}\text { Reported } \\
\text { effect size } \\
\text { (random } \\
\text { metatrim), } \\
\%(95 \% \mathrm{Cl})\end{array}$ & $\begin{array}{l}\text { Adjusted effect size } \\
\text { (random), } \\
\%(95 \% \mathrm{Cl})\end{array}$ & $\begin{array}{l}\text { Filled experiments } \\
\text { (was), } n\end{array}$ & $\begin{array}{l}\text { Absolute overestimation of } \\
\text { efficacy, \% }\end{array}$ & $\begin{array}{l}\text { Detectable bias with } \\
\text { Egger test? }\end{array}$ \\
\hline Decompression & $27.1(15.1-39.1)$ & $12.8(1.7$ to 24.0$)$ & $9(22)$ & 14.3 (-2.1 to 30.7$)$ & $+++(p<0.001)$ \\
\hline Exercise & $14.4(6.4-22.3)$ & $1.9(-6.9$ to 10.7$)$ & $7(35)$ & 12.5 (0.7 to 24.3$)$ & $-(p=0.72)$ \\
\hline Hypothermia & $16.3(10.3-22.3)$ & 16.3 & $-(11)$ & - & $-(p=0.799)$ \\
\hline OEG & $20.1(15.2-24.9)$ & 19.2 (14.4 to 24.0$)$ & $1(53)$ & 0.9 (-5.9 to 7.7$)$ & $+++(p<0.001)$ \\
\hline $\begin{array}{l}\text { Rho/ROCK } \\
\text { inhibitors }\end{array}$ & $21.3(16.0-26.6)$ & 15.6 (10.1 to 21.2 ) & $9(30)$ & $5.7(-2.0$ to 13.4$)$ & $+++(p<0.001)$ \\
\hline Stem cells & $26.2(23.9-28.5)$ & 26.2 & $-(278)$ & - & $-(p=0.12)$ \\
\hline
\end{tabular}

Abbreviations: BBB = Basso, Beattie, and Bresnahan; BMS = Basso Mouse Scale; CI = confidence interval; OEG = olfactory ensheathing glia. Different interventions were investigated for the inherent calculated number of reporting bias (missing experiments) and absolute overstatement of efficacy (delta inflation) by Duval and Tweedie nonparametric trim and fill analysis. This provides an estimate of the number of unpublished studies and an estimate of what the observed efficacy might have been had these studies been available. When no adjustment is made, either there are not enough data to infer the number of missing studies or there is no publication bias. Egger regression was added as an additional test to detect whether missing data reached significant levels (right column). Analyses were calculated for each singular intervention.

\section{Location of the laboratory}

The regional location of the experimental laboratory affected the reported effect size (figure 2E). Most of the animal experiments were performed in China, the United States, and Japan $\left(R^{2}=8.3 \%, p<0.0001\right)$. Studies from China and Australia were associated with large effect sizes, while studies from Brazil, Sweden, the United States, and Canada were associated with the smallest effect sizes. The required number of total animals for each listed country was 200, excluding those otherwise assigned to others.

\section{Time point of assessment}

Studies including long-term outcomes $(>2$-month assessment time frame) were underrepresented. The effect size of the investigated intervention decreased with extended assessment period after SCI, starting to intermittently drop below the average with observational time frames $>8$ weeks. Declining effect sizes after 8 weeks point to the risk that interventions provided only transient benefits, diminishing with time and therefore unlikely to translate into long-term results. This is in line with interventions that are able to trigger faster recovery but fail to reach higher functional plateaus at longer follow-up. The pattern of diminished effect size was not dependent on early vs late intervention. This rules out the possibility that decreasing effect sizes after 8 weeks resulted from more studies testing delayed applications. Variability was the highest in cases when the observational time was not indicated $\left(R^{2}\right.$ $=4.6 \%, p=0.0017$; figure $2 \mathrm{~F}$ ).

\section{Detection of publication bias (missing data)}

When considering all studies of the homogeneous BBB/BMS only cohort (table 2) comprising data from 6,302 animals stratified for the type of intervention, we observed an overestimation of efficacy due to missing data. Funnel plotting and trim and fill analysis indicated that data were missing in 4 types of SCI interventions. The reported effect size in each study was adjusted for absolute overestimation of efficacy for each study ranging between $0.9 \%$ (OEG) and $14.3 \%$ (decompression) (figure 3 and table 2). Assessment of the missing data/publication bias with the trim and fill method suggested that the number of missing experiments varies among the tested interventions, ranging from $0 \%$ (stem cells, hypothermia) to $1.9 \%$ (OEG), $20 \%$ (exercise), 30\% (Rho/ ROCK inhibition), and $40.9 \%$ (decompression). Data asymmetry indicative of missing data was confirmed by Egger regression, suggesting significant bias in the $\mathrm{BBB} / \mathrm{BMS}$ only datasets.

\section{Geographic differences}

To explore the differences attributable to the region-specific effect sizes (table 3), we focused on a comparison of the 3 countries with high study numbers and comparably small CIs (Unites States, Japan, and China). We excluded data from other countries with considerably lower study numbers (indicated by smaller bar width, figure $2 \mathrm{E}$ ) because the analysis would have been underpowered. Between-country differences may be related to varying percentages of interventions associated with larger effect sizes (percentage of stem cell studies: China 31.3\%, Japan 25.5\%, and United States 17.3\%). However, when we focused on stem cell interventions only, a similar geographic pattern was observed, with the highest effect size (31.3\%) observed in studies from Chinese laboratories compared to studies from Japan $(25.5 \%)$ or the United States (17.3\%). When we compared the effect sizes of recovery after complete transection as a defined injury type, the response to treatment was highest in studies from China (28.2\%), followed by Japan (9.3\%) and the United States (8.5\%). An association of poor study quality (characterized by the quality score) with larger therapeutic effect sizes has been reported earlier in other CNS lesion paradigms and is 

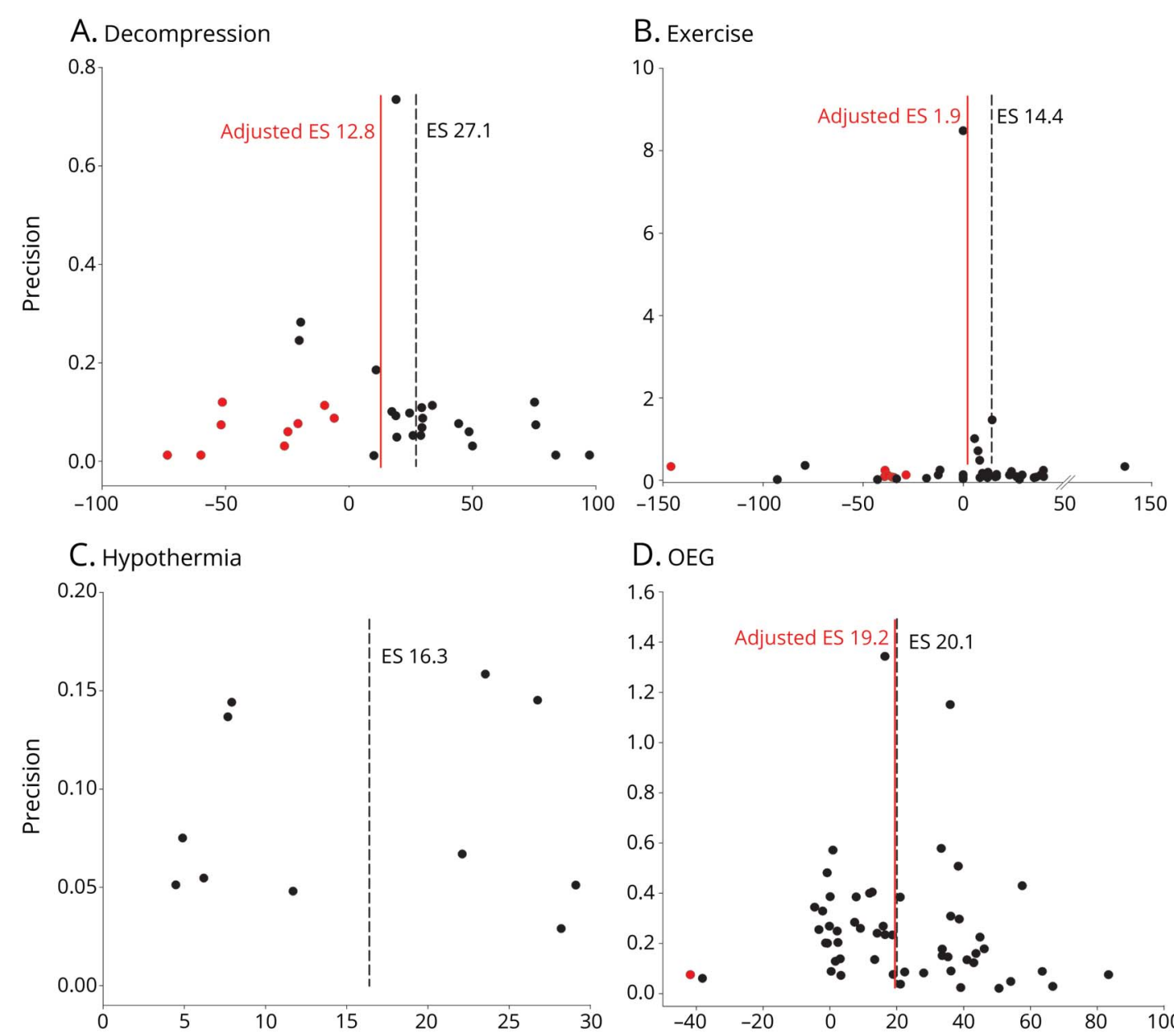

D. OEG
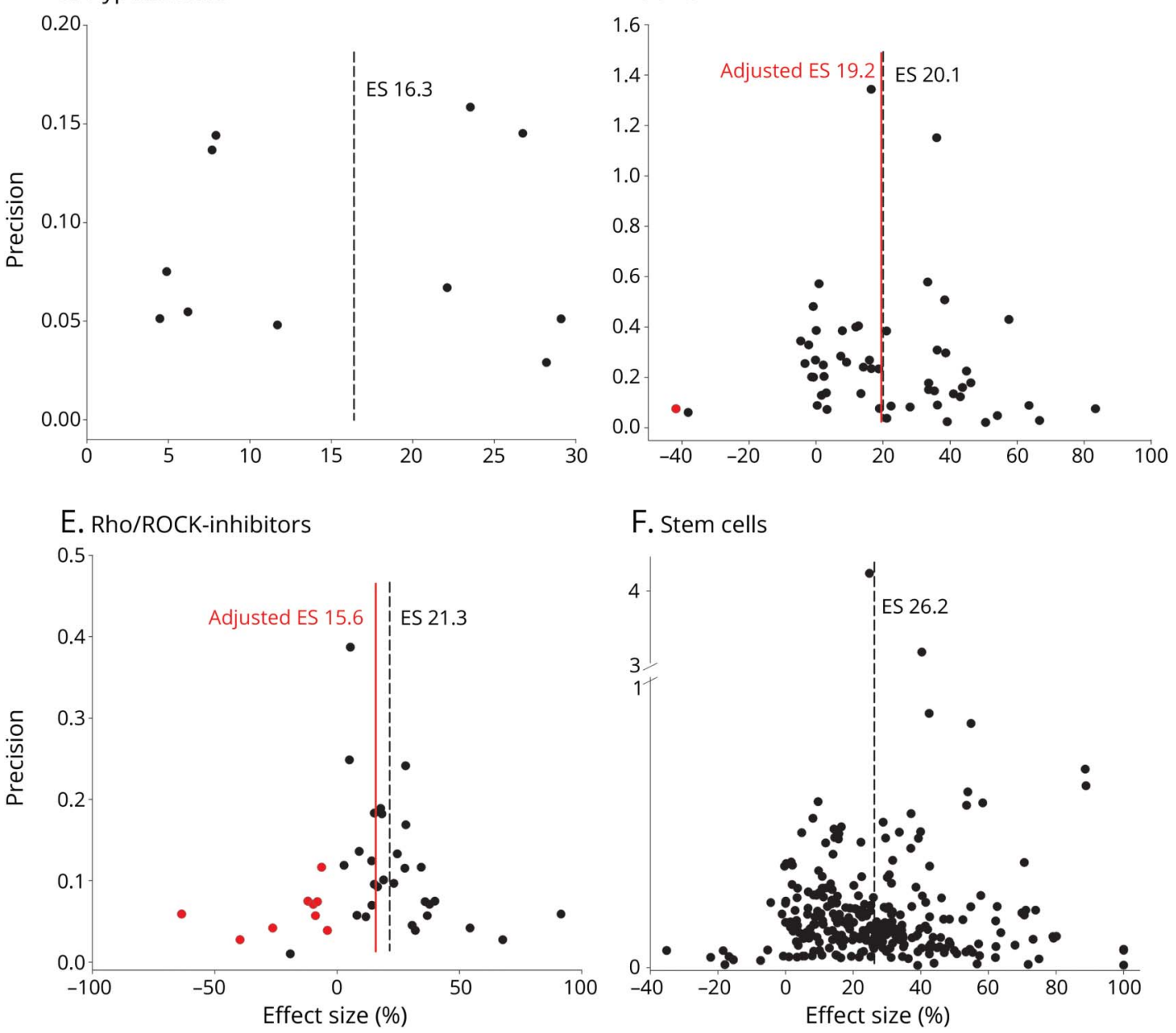

F. Stem cells

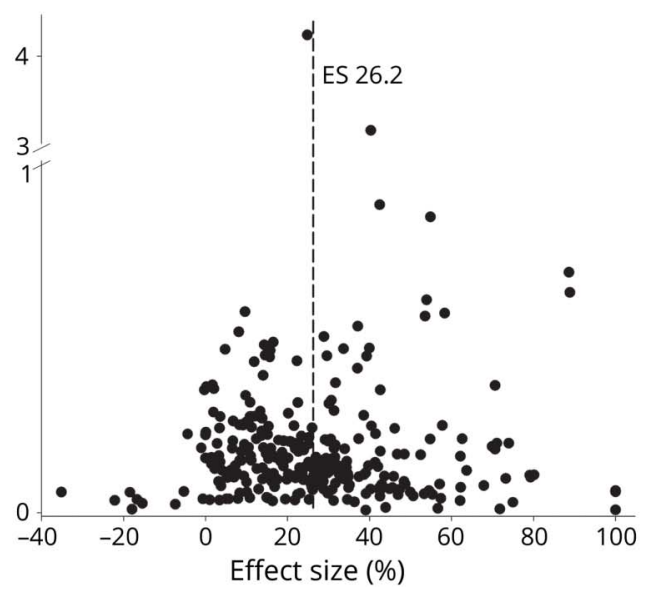

To allow a more homogeneous group characterized by similar effect sizes (ESs), a rodent analysis was completed identifying the Basso, Beattie, and Bresnahan (BBB)/Basso Mouse Scale (BMS) only group as comparable with regard to the main outcome parameter (improved locomotor function, figure 1B, red square). Funnel plots illustrating the precision (1 divided by the SEM, y-axis) plotted against the standardized ES (x-axis). Each black dot represents 1 experiment. In the absence of publication bias, the points should resemble an inverted funnel. Black dotted line indicates the reported ES before inclusion of missing studies; red line, after trim and till analysis. (A) Decompression: reported ES 27.1\% (95\% confidence interval [CI] 15.1-39.1, $\mathrm{n}=22$ studies), adjusted ESs 12.8\% (95\% Cl 1.7-24.0, $\mathrm{n}=31)$. (B) Exercise: reported ES 14.4\% (95\% Cl 6.4-22.3, $\mathrm{n}=35$ ), adjusted ES 1.9\% (95\% Cl -6.9 to 10.7, $\mathrm{n}=42)$. (C) Hypothermia: reported ES 16.3\% (95\% Cl 10.3-22.3) without adjustments. (D) Olfactory ensheathing glia (OEG): reported ES 20.1\% (95\% Cl 15.2-24.9, $n=53)$, adjusted ES 19.2 ( $95 \% \mathrm{Cl} 14.4-24.0, n=54)$. (E) Rho/ROCK inhibitors: reported ES 21.3\% (95\% Cl 16.0-26.6, $n=30)$, adjusted ES 15.6\% (95\% Cl 10.1-21.2, $n=39)$. (F) Stem cells: reported ES $26.2 \%(95 \% \mathrm{Cl} 23.9-28.5)$ without detection of missing studies. 
Table 3 Study characteristics of the BBB/BMS subanalysis based on geography

\begin{tabular}{|c|c|c|c|c|c|c|c|c|c|c|c|c|}
\hline & \multicolumn{4}{|c|}{ China $(n=2,177)$} & \multicolumn{4}{|c|}{ Japan $(n=960)$} & \multicolumn{4}{|c|}{ United States $(n=2,039)$} \\
\hline & ES & $95 \% \mathrm{Cl}$ & No. & $p$ Value & ES & $95 \% \mathrm{Cl}$ & No. & $p$ Value & ES & $95 \% \mathrm{Cl}$ & No. & $p$ Value \\
\hline \multicolumn{13}{|l|}{ Quality score } \\
\hline 1 & 22.5 & 4.4 to 40.6 & 89 & 0.53 & 49.1 & 33.8 to 64.4 & 132 & 0.002 & 38.7 & 14.3 to 63.1 & 112 & 0.06 \\
\hline 2 & 29.4 & 18.7 to 40.1 & 395 & & 32.0 & 20.1 to 43.9 & 250 & & 18.3 & 2.6 to 34 & 362 & \\
\hline 3 & 37.2 & 30.8 to 43.7 & 613 & & 16.1 & 8.9 to 23.4 & 183 & & 17.9 & 6.4 to 29.3 & 425 & \\
\hline 4 & 30.4 & 21 to 39.8 & 613 & & 23.0 & -0.2 to 46.1 & 78 & & 11.8 & -4 to 27.7 & 369 & \\
\hline 5 & 29.6 & 19.7 to 39.5 & 395 & & 18.6 & 7.3 to 29.9 & 247 & & 4.0 & -12.2 to 20.2 & 437 & \\
\hline 6 & 27.4 & 2.6 to 52.2 & 52 & & 18.6 & 0.7 to 36.5 & 70 & & 22.3 & 5.9 to 38.8 & 334 & \\
\hline 7 & 22.9 & -16.9 to 62.8 & 20 & & - & & & & - & & & \\
\hline \multicolumn{13}{|l|}{ Type of SCI } \\
\hline Compression & 35.0 & 22.8 to 47.1 & 336 & 0.35 & 21.5 & 7.1 to 35.8 & 117 & 0.37 & 10.5 & -33.5 to 54.5 & 45 & 0.26 \\
\hline Hemisection & 33.9 & 22.8 to 45 & 367 & & 19.6 & -1.6 to 40.7 & 109 & & 30.6 & 13.6 to 47.6 & 330 & \\
\hline Transection & 28.2 & 19.8 to 36.5 & 813 & & 9.3 & -12.3 to 30.9 & 45 & & 8.5 & -10.4 to 27.3 & 172 & \\
\hline Contusion & 34.7 & 28 to 41.3 & 661 & & 26.0 & 20.1 to 31.9 & 689 & & 15.2 & 9.6 to 20.7 & 1,492 & \\
\hline
\end{tabular}

Abbreviations: $\mathrm{BBB}=$ Basso, Beattie, and Bresnahan; BMS = Basso Mouse Scale; $\mathrm{Cl}$ = confidence interval; $\mathrm{ES}$ = effect size; $\mathrm{SCl}=$ spinal cord injury .

For a more detailed analysis on varying effect sizes dependent on the location of the publishing laboratory, we focused on countries with a robust number of experiments. Representative countries include Japan ( $n=960$ rodents), the United States $(n=2,039)$, and China $(n=2,177)$. This analysis corresponds to the overall findings displayed in figure $2 \mathrm{E}$, suggesting regional effects with regard to the reporting of study results.

observable in the SCI studies from Japan $(p=0.002)$ and the United States $(p=0.06)$. In contrast, in studies from China, the effect size did not correlate with the quality score.

\section{Discussion}

We demonstrate the feasibility and utility of a metaepidemiologic approach for systematic assessment of outcome variability and detection of the presence of bias in the design and reporting of experimental SCI studies through a meta-analysis of data from 303 publications including 9,535 experimental animals. We provide empirical evidence for bias in experimental SCI research resulting in inflated effect sizes, and we report the effect of study design characteristics on heterogeneity of neurobehavioral recovery.

Rodent females constitute the largest population used in preclinical SCI models, with rats outnumbering mice (figures 1 and 2). The analysis of outcome measures confirms openfield $\mathrm{BBB}$ and $\mathrm{BMS}$ testing as robust measures of locomotor recovery characterized by comparable effect sizes. The data reemphasize the evidence that a combination of multiple functional outcome measures examining particular components of recovered motor function results in the reporting of less inflated effect sizes. ${ }^{32}$ Nonhuman primates were associated with a large effect size and variability. The Tarlov score was developed in $1954^{25}$ and is outdated.
Independent group assignment (blinded SCI induction, allocation concealment) and blinded assessment (masking) of outcome are crucial to avoid prevalent selection and evaluation bias; more than half of the studies analyzed lacked blinding. Lack of blinding was associated with a significant overstatement of efficacy by $7.2 \%$ in the overall cohort when considering all species and interventions. However, relative to the effect size of tested interventions, up to $55 \%$ of interventional effect may be inflated and attributable to the lack of blinding (e.g., $7.2 \%$ of $13.1 \%$ for exercise as an intervention). In addition to blinding, compliance with regulatory requirements is considered a measure of study quality. ${ }^{21}$ Lack of reporting of this criterion was associated with an overstatement of efficacy by $5.6 \%$.

To provide a more homogeneous subcohort for studying differential effects of intervention type, lesion level, sex, injury modality, geographic location of publishing laboratory, effect durability, and missing data (publication/reporting bias), the subsequent analysis was restricted to a rodent $\mathrm{BBB} / \mathrm{BMS}$ only cohort $(n=7,613)$.

\section{Interventions}

We identified 6 different therapeutic interventions, including the spectrum of cellular therapies (stem cells, OEG), systemically applied approaches (hypothermia, Rho/ROCK inhibitors), and rehabilitative (exercise) and surgical interventions (decompression). Four of 6 interventions (67\%) were characterized by data asymmetry, attributable largely to 
missing data. For 3 of 6 interventions (50\%), our analysis identified an overstatement of efficacy (table 2). The effect sizes of classic acute care interventions (decompressive surgery and hypothermia) were larger than the effect sizes of rehabilitation interventions. Effects of exercise interventions display a large $\mathrm{CI}$ and point to the existence of variable factors determining rehabilitation efficacy. These results also suggest that rehabilitation cannot fully compensate for what has been lost during acute care and could emphasize the therapeutic value of optimal specialized acute SCI care. Retrospective clinical studies of acute care interventions such as decompressive surgery support this notion. ${ }^{34,35}$

\section{$\mathrm{SCI}$ lesion level}

We provide evidence that in addition to varying lesion severity, the distance from the lesion to the lumbosacral motor pools is an important determinant of responsiveness to treatment (figure $2 \mathrm{~B}$ ). The more distant the lesion is, the lower the neurologic recovery is. The increase of effect size per spinal level, descending along the neuraxis, is not linear. The effect size per lesion level distal to Th10 results in a remarkable increased step of effect sizes. This stratification confirmed the biological plausibility of the SCI model, with increasing effect size the closer the lesion is located to lumbosacral motor pools, suggesting the propagation of intrinsic repair/regeneration pathways as the underlying mechanism of recovery. Less severe high cervical models will naturally allow higher compensatory plasticity, contributing to larger effect sizes, which render them mechanistically noncomparable to complete transection or contusion SCI applied to low cervical to low thoracic sites.

\section{Sex}

Female rodents displayed smaller effect sizes compared to males (figure 2C). Thoracic SCI is the most prevalent cohort modeling experimental SCI (figure 2B), matching to some degree with lower functional recovery in women after thoracic (T2-8) SCI. However, this is only a transient effect and is no longer detectable 1 year after human SCI. ${ }^{36}$

\section{Injury type}

Contusion injury was the most severe injury, characterized by smallest effect sizes in response to interventional therapy. This is consistent with evidence of injury exacerbation extending both rostrally and caudally from the lesion site after contusion, whereas even after complete transection, injury remains localized to the initial lesion site. ${ }^{32,37}$ Static clip compression injury is a slightly less severe SCI paradigm (figure 2D).

\section{Geographic effects}

Stratification for the location of publishing laboratory revealed differences (figure 2E). Study results from Japan and the United States showed an inverse association between effect sizes and quality, confirming findings from a previous metaanalysis in animal research, ${ }^{21}$ whereas studies from China did not (table 3). Chinese studies demonstrated smaller variability of outcomes compared to studies from Japan and the United States. Larger effect sizes concomitant with smaller CIs are consistent with the suggestion that animals considered to be outliers have been removed from further analysis, contributing to attrition bias. ${ }^{38}$ The data suggest geographic differences with regard to the reporting of study results and emphasize the importance of reporting attrition and its reasons.

\section{Unstable (transient) treatment effects}

We observed an intermittent decline of effect sizes from 8 weeks after SCI on, suggesting the presence of concomitant processes that undermine gained locomotor functions. Several processes may account for this, including delayed neurodegeneration ${ }^{39}$ and salutatory nerve conduction failure of intact axons at the chronic lesion site. ${ }^{40,41}$ Our results suggest that an observational time frame of $\geq 8$ weeks is required to report durable effects. The data indicate a risk for transient interventional effects and the need to favor long-term over short-term outcome measures, in line with other models of acute CNS injury. ${ }^{42}$

\section{Reporting bias (missing data)}

We provide empirical evidence of missing data. Trim and fill analysis of the entire BBB/BMS only cohort identified missing data. This was confirmed by Egger regression (table 2). Publication bias is a fundamental problem in preclinical research across many disciplines. ${ }^{30}$ The presence of such bias leads to inflated effect sizes and skewed biometric planning of clinical trials and jeopardizes the successful translation of SCI therapies from bench to bedside. The fact that for some of the interventions no publication bias could be detected may have several reasons and does not automatically imply that it is not present at all. The analysis of interventions with a small number of experiments is most likely underpowered. In addition, even for interventions with large sample sizes, the precision of experiments may be falsely increased due to unexplored attrition.

Regarding limitations of this study, we were able to include only data from studies in the public domain. Second, for both study quality and study design characteristics, we relied on published information. When relevant data were not available (the sex of a cohort of animals or measures taken to reduce bias), we have either analyzed them as not known or inferred that measures that were not reported did not occur. Third, we present a series of univariate meta-regression analyses. Multivariate meta-regression or stepwise partitioning of heterogeneity might provide more robust insights, but these techniques are not well established today. Fourth, our analysis depended on the validity of the scales used, which has been questioned. ${ }^{43}$ Fifth, translation into human SCI might be dampened by limitations of the animal SCI model, affecting the external validity, such as the absence of polytrauma (including vertebrae fractures). Finally, we accept the weakness of the presented meta-analysis, which combines various animal models of specific SCI treatments within a very large 
dataset and assumes that random-effect meta-regression can account for this. Detecting the likelihood of preventable bias is still a comparatively novel but imperfect science because reporting standards are generally poor. However, while imperfect, the analysis represents best evidence available, representing one of the largest studies on SCI animal models to date.

In this study, we provide empirical evidence for differential outcome characteristics attributable to distinct SCI models and experimental designs. We observed evidence for transient treatment effects, as well as evaluation and publication bias, which diminish the experimental validity and could cause clinical trials to follow false leads. The presence of publication bias in preclinical SCI research confirms the endemic presence of underlying bias in science, ${ }^{11}$ rather than being specific to SCI. ${ }^{29}$ Bias in animal SCI experiments erodes the predictive value for translation into human SCI trials. Bias is not restricted to experimental testing but also occurs later in the translational spectrum in early clinical testing. ${ }^{44}$

The SCI field is proactively responding to this challenge. ${ }^{45}$ Our data inform 4 recommendations to enhance the predictive value of experimental SCI research for a higher likelihood of translational success:

1. Reduce bias at the benchside ${ }^{46}$ through allocation concealment and blinded assessment of outcome; by reporting animals excluded from the analysis ${ }^{38}$; and by providing a sample size calculation, inclusion and exclusion criteria, randomization, and transparent reporting of potential conflicts of interest and study funding. ${ }^{12,42}$

2. Report nonconfirmatory (negative or neutral) results, which are persistently underrepresented in the public domain.

3. Use international experimental and reporting standards in preclinical SCI studies according to the Minimum Information About a Spinal Cord Injury Experiment ${ }^{47}$ or Animal Research: Reporting of In Vivo Experiments guidelines ${ }^{48}$ to reduce study design variability.

4. Develop data-sharing models ${ }^{49-51}$ and international cooperation in preclinical SCI research to learn more about underlying differences.

\section{Author contributions}

Conception and design of the study: R. Watzlawick, E. Sena, M. Macleod, D.W. Howells, J.M. Schwab; acquisition and analysis of data: R. Watzlawick, A. Antonic, J. Rind; drafting a significant portion of the manuscript or figures: $R$. Watzlawick, A. Antonic, M.A. Kopp, U. Dirnagl, D.W. Howells, J.M. Schwab.

\section{Acknowledgment}

The authors thank Gillian Currie for expert technical assistance. J.M.S. had full access to all the data in the study and takes responsibility for the integrity of the data and the accuracy of the data analysis. The Department of Clinical and Experimental Spinal Cord Injury Research is an associated member of the European Multicenter Study About Spinal Cord Injury.

\section{Study funding}

No targeted funding reported.

\section{Disclosure}

R. Watzlawick, A. Antonic, E. Sena, M. Kopp, J. Rind, U. Dirnagl, M. Macleod, and D. Howells report no disclosures relevant to the manuscript. J. Schwab received funding support from the Else-Kroehner-Fresenius Foundation (No. 2012_A32); the Wings-for-Life Spinal Cord Research Foundation (Nos. DE-047/14, DE-16/16); the Era-NetNEURON Program of the European Union (SILENCE No. 01EW170A, SCI-Net No. 01EW1710); the National Institute on Disability, Independent Living, and Rehabilitation Research (No. 90SI5020); and the W.E. Hunt \& C.M. Curtis Endowment. J.M.S. is a Discovery Theme Initiative Scholar (Chronic Brain Injury) of The Ohio State University. Go to Neurology.org/ $\mathrm{N}$ for full disclosures.

\section{Publication history}

Received by Neurology August 27, 2018. Accepted in final form February 11, 2019.

\section{References}

1. Ahuja CS, Wilson JR, Nori S, et al. Traumatic spinal cord injury. Nat Rev Dis Primers 2017;3:17018

2. Schwab JM, Brechtel K, Mueller CA, et al. Experimental strategies to promote spinal cord regeneration: an integrative perspective. Prog Neurobiol 2006;78:91-116.

3. Jain NB, Ayers GD, Peterson EN, et al. Traumatic spinal cord injury in the United States, 1993-2012. JAMA 2015;313:2236-2243.

4. Metz GA, Curt A, van de Meent H, Klusman I, Schwab ME, Dietz V. Validation of the weight-drop contusion model in rats: a comparative study of human spinal cord injury. J Neurotrauma 2000;17:1-17.

5. Dietz V, Curt A. Neurological aspects of spinal-cord repair: promises and challenges. Lancet Neurol 2006;5:688-694.

6. Courtine G, Bunge MB, Fawcett JW, et al. Can experiments in nonhuman primates expedite the translation of treatments for spinal cord injury in humans? Nat Med 2007; 13:561-566.

7. Friedli L, Rosenzweig ES, Barraud Q, et al. Pronounced species divergence in corticospinal tract reorganization and functional recovery after lateralized spinal cord injury favors primates. Sci Transl Med 2015;7:302ra134.

8. Sjovold SG, Mattucci SF, Choo AM, et al. Histological effects of residual compression sustained for 60 minutes at different depths in a novel rat spinal cord injury contusion model. J Neurotrauma 2013;30:1374-1384.

9. Seok J, Warren HS, Cuenca AG, et al. Genomic responses in mouse models poorly mimic human inflammatory diseases. Proc Natl Acad Sci USA 2013;110:3507-3512.

10. Howells DW, Sena ES, Macleod MR. Bringing rigour to translational medicine. Nat Rev Neurol 2014;10:37-43.

11. Landis SC, Amara SG, Asadullah K, et al. A call for transparent reporting to optimize the predictive value of preclinical research. Nature 2012;490:187-191.

12. Macleod MR, van der Worp HB, Sena ES, Howells DW, Dirnagl U, Donnan GA. Evidence for the efficacy of NXY-059 in experimental focal cerebral ischaemia is confounded by study quality. Stroke 2008;39:2824-2829.

13. Button KS, Ioannidis JP, Mokrysz C, et al. Power failure: why small sample size undermines the reliability of neuroscience. Nat Rev Neurosci 2013;14:365-376.

14. Ioannidis JP. Why most published research findings are false. PLoS Med 2005;2:e124

15. Antonic A, Sena ES, Lees JS, et al. Stem cell transplantation in traumatic spinal cord injury: a systematic review and meta-analysis of animal studies. PLoS Biol 2013;11: e1001738.

16. Batchelor PE, Skeers P, Antonic A, et al. Systematic review and meta-analysis of therapeutic hypothermia in animal models of spinal cord injury. PLoS One $2013 ; 8$ : e71317.

17. Batchelor PE, Wills TE, Skeers P, et al. Meta-analysis of pre-clinical studies of early decompression in acute spinal cord injury: a battle of time and pressure. PLoS One 2013;8:e72659. 
18. Battistuzzo CR, Callister RJ, Callister R, Galea MP. A systematic review of exercise training to promote locomotor recovery in animal models of spinal cord injury. J Neurotrauma 2012;29:1600-1613.

19. Watzlawick R, Rind J, Sena ES, et al. Olfactory ensheathing cell transplantation in experimental spinal cord injury: effect size and reporting bias of 62 experimental treatments: a systematic review and meta-analysis. PLoS Biol 2016;14:e1002468.

20. Watzlawick R, Sena ES, Dirnagl U, et al. Effect and reporting bias of RhoA/ROCKblockade intervention on locomotor recovery after spinal cord injury: a systematic review and meta-analysis. JAMA Neurol 2014;71:91-99.

21. Macleod MR, O'Collins T, Howells DW, Donnan GA. Pooling of animal experimental data reveals influence of study design and publication bias. Stroke 2004;35:1203-1208.

22. Vesterinen HM, Sena ES, Egan KJ, et al. Meta-analysis of data from animal studies: a practical guide. J Neurosci Methods 2014;221:92-102.

23. Basso DM, Beattie MS, Bresnahan JC. A sensitive and reliable locomotor rating scale for open field testing in rats. J Neurotrauma 1995;12:1-21.

24. Basso DM, Fisher LC, Anderson AJ, Jakeman LB, McTigue DM, Popovich PG. Basso Mouse Scale for locomotion detects differences in recovery after spinal cord injury in five common mouse strains. J Neurotrauma 2006;23:635-659.

25. Tarlov IM. Spinal cord compression studies. III: time limits for recovery after gradual compression in dogs. AMA Arch NeurPsych 1954;71:588-597.

26. Harbord RM, Higgins JPT. Meta-regression in Stata. Stata J 2008;8:493-519.

27. Jackson D, Riley R, White IR. Multivariate meta-analysis: potential and promise. Stat Med 2011;30:2481-2498.

28. Light RJ, Pillemer DB. Summing up: The Science of Reviewing Research. Cambridge: Harvard University Press; 1984.

29. Duval S, Tweedie R. Trim and fill: a simple funnel-plot-based method of testing and adjusting for publication bias in meta-analysis. Biometrics 2000;56:455-463.

30. Sena ES, van der Worp HB, Bath PM, Howells DW, Macleod MR. Publication bias in reports of animal stroke studies leads to major overstatement of efficacy. PLoS Biol 2010;8:e1000344.

31. Egger M, Davey Smith G, Schneider M, Minder C. Bias in meta-analysis detected by a simple, graphical test. BMJ 1997;315:629-634.

32. Kwon BK, Oxland TR, Tetzlaff W. Animal models used in spinal cord regeneration research. Spine 2002;27:1504-1510.

33. Sroga JM, Jones TB, Kigerl KA, McGaughy VM, Popovich PG. Rats and mice exhibit distinct inflammatory reactions after spinal cord injury. J Comp Neurol 2003;462:223-240.

34. Fehlings MG, Vaccaro A, Wilson JR, et al. Early versus delayed decompression for traumatic cervical spinal cord injury: results of the Surgical Timing in Acute Spinal Cord Injury Study (STASCIS). PLoS One 2012;7:e32037.

35. Wilson JR, Singh A, Craven C, et al. Early versus late surgery for traumatic spinal cord injury: the results of a prospective Canadian cohort study. Spinal Cord 2012;50: $840-843$.
36. Sipski ML, Jackson AB, Gómez-Marín O, Estores I, Stein A. Effects of gender on neurologic and functional recovery after spinal cord injury. Arch Phys Med Rehabil 2004;85:1826-1836.

37. Siegenthaler MM, Tu MK, Keirstead HS. The extent of myelin pathology differs following contusion and transection spinal cord injury. J Neurotrauma 2007;24: 1631-1646.

38. Holman C, Piper SK, Grittner U, et al. Where have all the rodents gone? The effects of attrition in experimental research on cancer and stroke. PLoS Biol 2016;14:e1002331.

39. Bramlett HM, Dietrich WD. Progressive damage after brain and spinal cord injury: pathomechanisms and treatment strategies. Prog Brain Res 2007;161:125-141.

40. Arvanian VL, Schnell L, Lou L, et al. Chronic spinal hemisection in rats induces a progressive decline in transmission in uninjured fibers to motoneurons. Exp Neurol 2009;216:471-480.

41. James ND, Bartus K, Grist J, Bennett DL, McMahon SB, Bradbury EJ. Conduction failure following spinal cord injury: functional and anatomical changes from acute to chronic stages. J Neurosci 2011;31:18543-18555.

42. Dirnagl U, Endres M. Found in translation: preclinical stroke research predicts human pathophysiology, clinical phenotypes, and therapeutic outcomes. Stroke 2014;45: 1510-1518.

43. Ferguson AR, Hook MA, Garcia G, Bresnahan JC, Beattie MS, Grau JW. A simple post hoc transformation that improves the metric properties of the BBB scale for rats with moderate to severe spinal cord injury. J Neurotrauma 2004;21:1601-1613.

44. Gomes-Osman J, Cortes M, Guest J, Pascual-Leone A. A systematic review of experimental strategies aimed at improving motor function after acute and chronic spinal cord injury. J Neurotrauma 2016;33:425-438.

45. Steward O, Popovich PG, Dietrich WD, Kleitman N. Replication and reproducibility in spinal cord injury research. Exp Neurol 2012;233:597-605.

46. Macleod MR, Fisher M, O'Collins V, et al. Good laboratory practice: preventing introduction of bias at the bench. Stroke 2009;40:e50-52.

47. Lemmon VP, Ferguson AR, Popovich PG, et al. Minimum information about a spinal cord injury experiment: a proposed reporting standard for spinal cord injury experiments. J Neurotrauma 2014;31:1354-1361.

48. Kilkenny C, Browne WJ, Cuthill IC, Emerson M, Altman DG. Improving bioscience research reporting: the ARRIVE guidelines for reporting animal research. PLoS Biol 2010;8:e1000412.

49. Callahan A, Anderson KD, Beattie MS, et al. Developing a data sharing community for spinal cord injury research. Exp Neurol 2017;295:135-143.

50. Ferguson AR, Nielson JL, Cragin MH, Bandrowski AE, Martone ME. Big data from small data: data-sharing in the "long tail" of neuroscience. Nat Neurosci 2014;17: 1442-1447.

51. Nielson JL, Paquette J, Liu AW, et al. Topological data analysis for discovery in preclinical spinal cord injury and traumatic brain injury. Nat Commun 2015;6:8581. 


\section{Neurology}

\section{Outcome heterogeneity and bias in acute experimental spinal cord injury: A meta-analysis}

Ralf Watzlawick, Ana Antonic, Emily S. Sena, et al.

Neurology 2019;93;e40-e51 Published Online before print June 7, 2019

DOI 10.1212/WNL.0000000000007718

This information is current as of June 7, 2019

\section{Updated Information \& Services}

References

Subspecialty Collections

Permissions \& Licensing

Reprints including high resolution figures, can be found at: http://n.neurology.org/content/93/1/e40.full

This article cites 50 articles, 8 of which you can access for free at: http://n.neurology.org/content/93/1/e40.full\#ref-list-1

This article, along with others on similar topics, appears in the following collection(s):

Plasticity

http://n.neurology.org/cgi/collection/plasticity

Spinal cord trauma

http://n.neurology.org/cgi/collection/spinal_cord_trauma

Information about reproducing this article in parts (figures,tables) or in its entirety can be found online at:

http://www.neurology.org/about/about_the_journal\#permissions

Information about ordering reprints can be found online:

http://n.neurology.org/subscribers/advertise

Neurology ${ }^{\circledR}$ is the official journal of the American Academy of Neurology. Published continuously since 1951, it is now a weekly with 48 issues per year. Copyright (O 2019 American Academy of Neurology. All rights reserved. Print ISSN: 0028-3878. Online ISSN: 1526-632X.

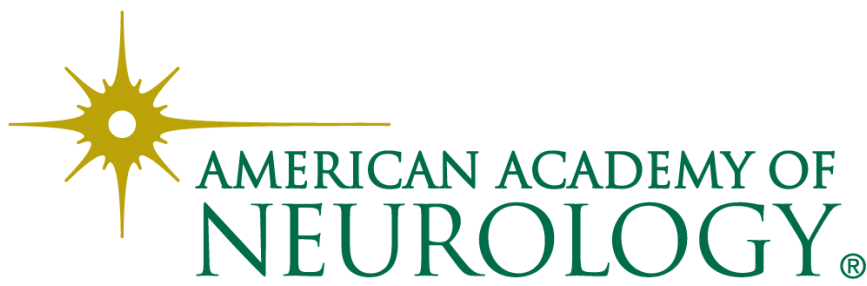

